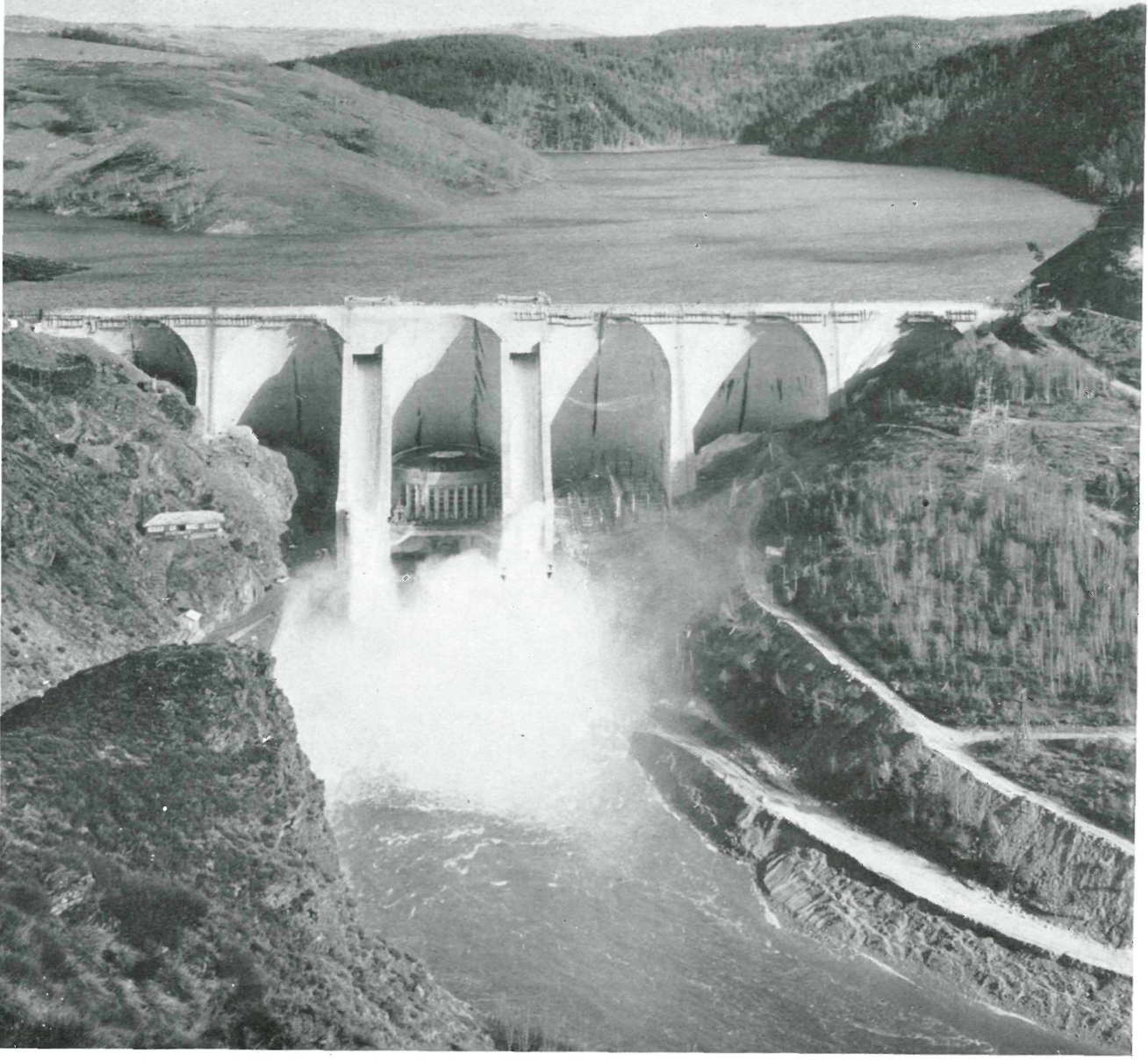

DIPESa de Grandval

C. LAROCHE Y H. LEGROS, ingenieros consultores del Gabinete Técnico Coyne y Bellier

$531-61$

\title{
sin opsis
}

Esta importante obra se halla situada en el tramo superior del río Trufrancés.

Las presas se han realizado por cuen ta de Electricité de France. El conjunto del aprovechamiento hidroeléctrico tiene seis presas y otras tantas centrales que aprovechan un salto total de $520 \mathrm{~m}$ de altura, $y$ las aguas almacenadas en an embalse cuya que proporcionarán una producción anual de $1.450 \times 10^{6} \mathrm{kWh}$. La presa apoya sobre un banco microesquistoso, que en algunas partes presenta grandes pliegues de gran potencia. El dique de cierre tiene una longitud total de $330 \mathrm{~m}$ y $88 \mathrm{~m}$ de altura máxima, a partir de la base de cimientos. Su estructura está constituida por una serie de bovedas que se apoyan en contrafuertes. La luz entre éstos es de 50 metros.
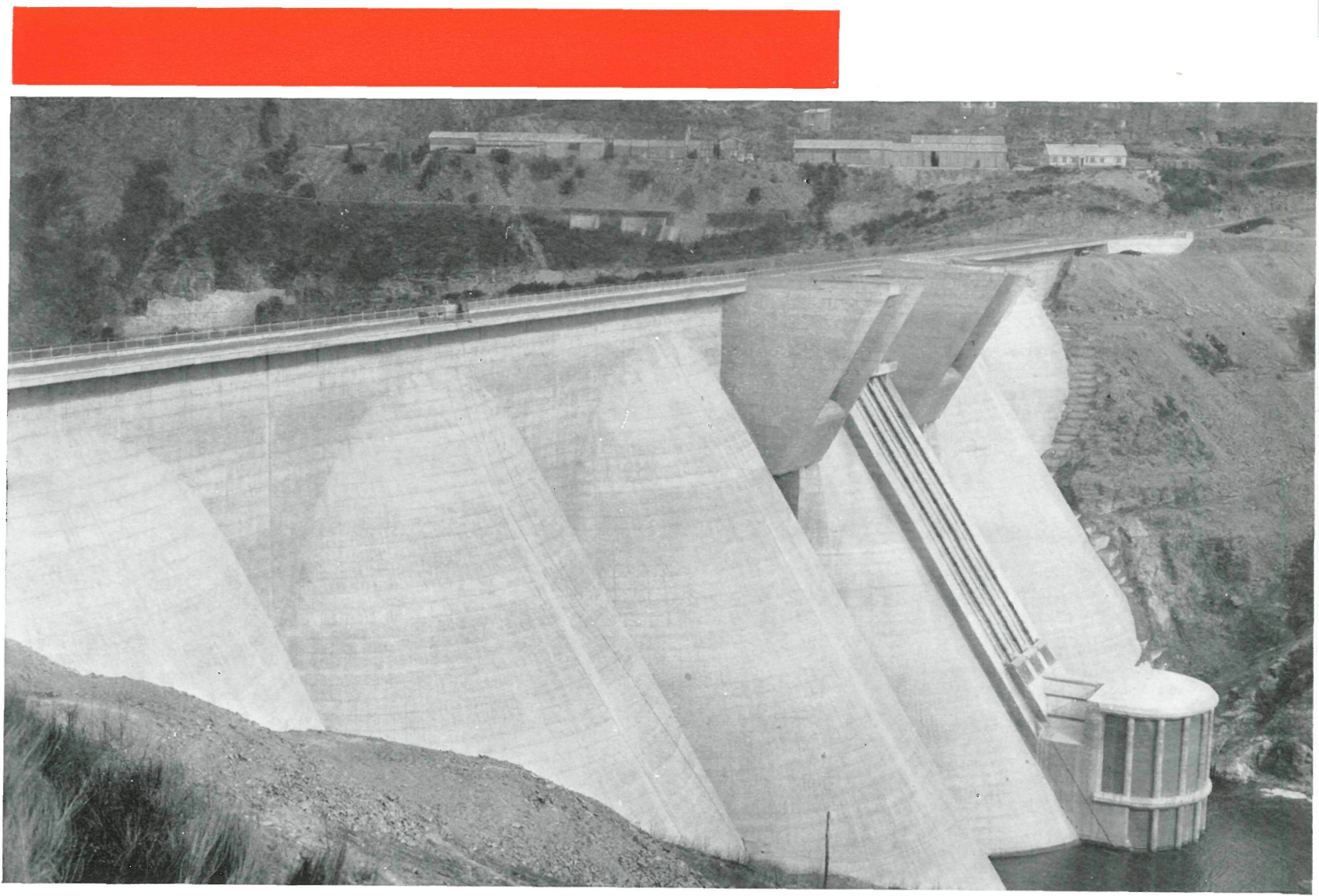


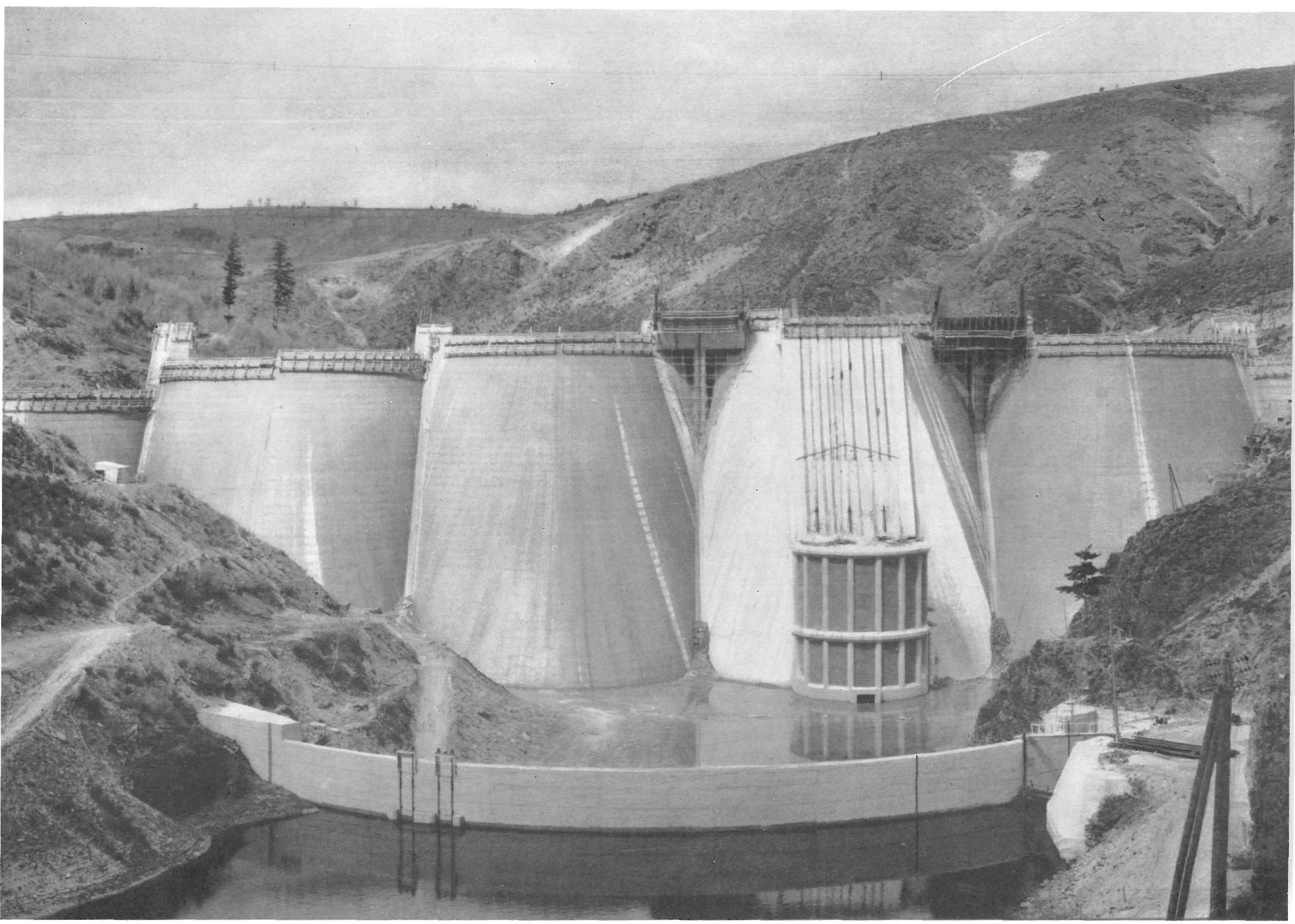

El espesor medio de las bóvedas es de $4,90 \mathrm{~m}$ en la base y de $1,50 \mathrm{~m}$ en coronación. El talud de aguas arriba es de $0,70 \times 1$, con la particularidad de que su construcción no tiene forma cilíndrica extendida hasta la coronación, como corrientemente se acostumbra. En la parte superior de estas bovedas se ha continuado, en elevación, formando un pequeño dique de gravedad. dispone de un aliviadero de superficie con compuerta de sector y vertedero en forma de salto de esquí sobre cada uno de los dos contrafuertes entre los que se ha construido la central.

Situación de la obra.-La presa Grandval está emplazada en el tramo superior del río Truyère, del Macizo Central (Francia).

El conjunto de este aprovechamiento hidroeléctrico, realizado por Electricité de France, consta de seis presas y seis centrales que aprovechan un salto hidrostático total de $520 \mathrm{~m}$ de altura y aportaciones medias del orden de $850 \times 16^{6} \mathrm{~m}$ por año para el embalse de Grandval y de $2.300 \times 10^{6} \mathrm{~m}^{3}$ para otros embalses. La capacidad total útil es de $540 \times 10^{6} \mathrm{~m}^{3}$, que corresponden a una producción media anual de $1.450 \times 10^{6} \mathrm{kWh}$

El embalse, propiamente dicho, de Grandval, tiene una capacidad útil de $239 \times 10^{6} \mathrm{~m}^{3}$, y alimenta a una central que dispone de $68.000 \mathrm{~kW}$ de potencia instalada y cuya productividad anual se eleva a $125 \times 10^{6} \mathrm{kWh}$

La cimentación se apoya en un terreno microesquistoso, de grandes pliegues, cubierto por una capa muy alterada y de bastante potencia en alguna de sus partes. En el lugar de ubicación se han llevado a cabo estudios experimentales sobre las características de esta roca, principalmente de su resistencia al esfuerzo cortante.

La presa.-La presa Grandval tiene una altura de $88 \mathrm{~m}$ respecto a la cota de cimentación y una longitud de $330 \mathrm{~m}$ en coronación. Está constituida por bóvedas múltiples de gran luz, las cuales alcanzan hasta unos 50 m entre ejes de contrafuertes.

La lámina de impermeabilización, constituida por las referidas bóvedas, trabaja en condiciones muy similares-en lo que a esfuerzos se refiere-a las de una bóveda de tipo ordinario. En lo que respecta a la presa de Grandval, su espesor, medido horizontalmente, es de 4,90 $\mathrm{m}$ en la base de la boveda más alta y de 1,50 $\mathrm{m}$ en coronación, proporcionando un espacio suficiente para poder hormigonar a un ritmo acelerado. Además, aun contando con un talud relativamente pronunciado, se ha podido prescindir de cimbras y entramados para sostener los encofrados del paramento de aguas abajo. Los utilizados han sido del tipo deslizante que, partiendo de la base, se han ido corriendo, en voladizo, a medida que la obra avanzaba. 

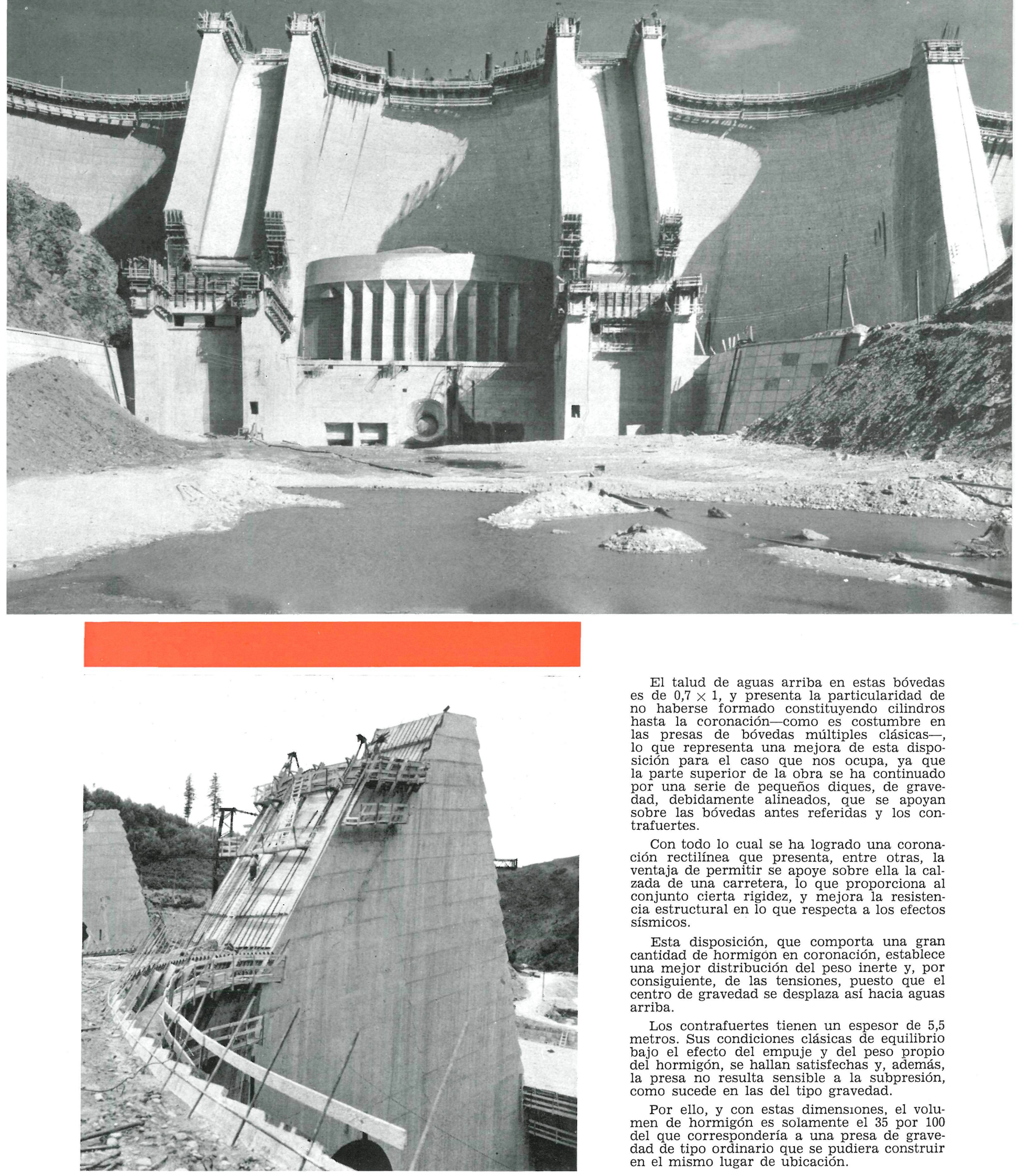

El talud de aguas arriba en estas bóvedas es de $0,7 \times 1$, y presenta la particularidad de no haberse formado constituyendo cilindros hasta la coronación-como es costumbre en las presas de bóvedas múltiples clásicaslo que representa una mejora de esta disposición para el caso que nos ocupa ya que la parte superior de la obra se ha continuado por una serie de pequeños diques, de gravedad, debidamente alineados, que se apoyan sobre las bóvedas antes referidas y los consobre las
trafuertes.

Con todo lo cual se ha logrado una coronación rectilínea que presenta, entre otras, la ventaja de permitir se apoye sobre ella la calzada de una carretera, lo que proporciona al conjunto cierta rigidez, y mejora la resistencia estructural en lo que respecta a los efectos sísmicos.

Esta disposición que comporta una gran cantidad de hormigon en coronación, establece una mejor distribución del peso inerte $\mathrm{y}$, por consiguiente, de las tensiones, puesto que el centro de gravedad se desplaza así hacia aguas arriba.

Los contrafuertes tienen un espesor de 5,5 metros. Sus condiciones clásicas de equilibrio bajo el efecto del empuje y del peso propio del hormigón, se hallan satisfechas y, además, la presa no resulta sensible a la subpresión, como sucede en las del tipo gravedad.

Por ello, y con estas dimensiones, el volumen de hormigón es solamente el 35 por 100 del que correspondería a una presa de gravedad de tipo ordinario que se pudiera construir en el mismo lugar de ubicación. 


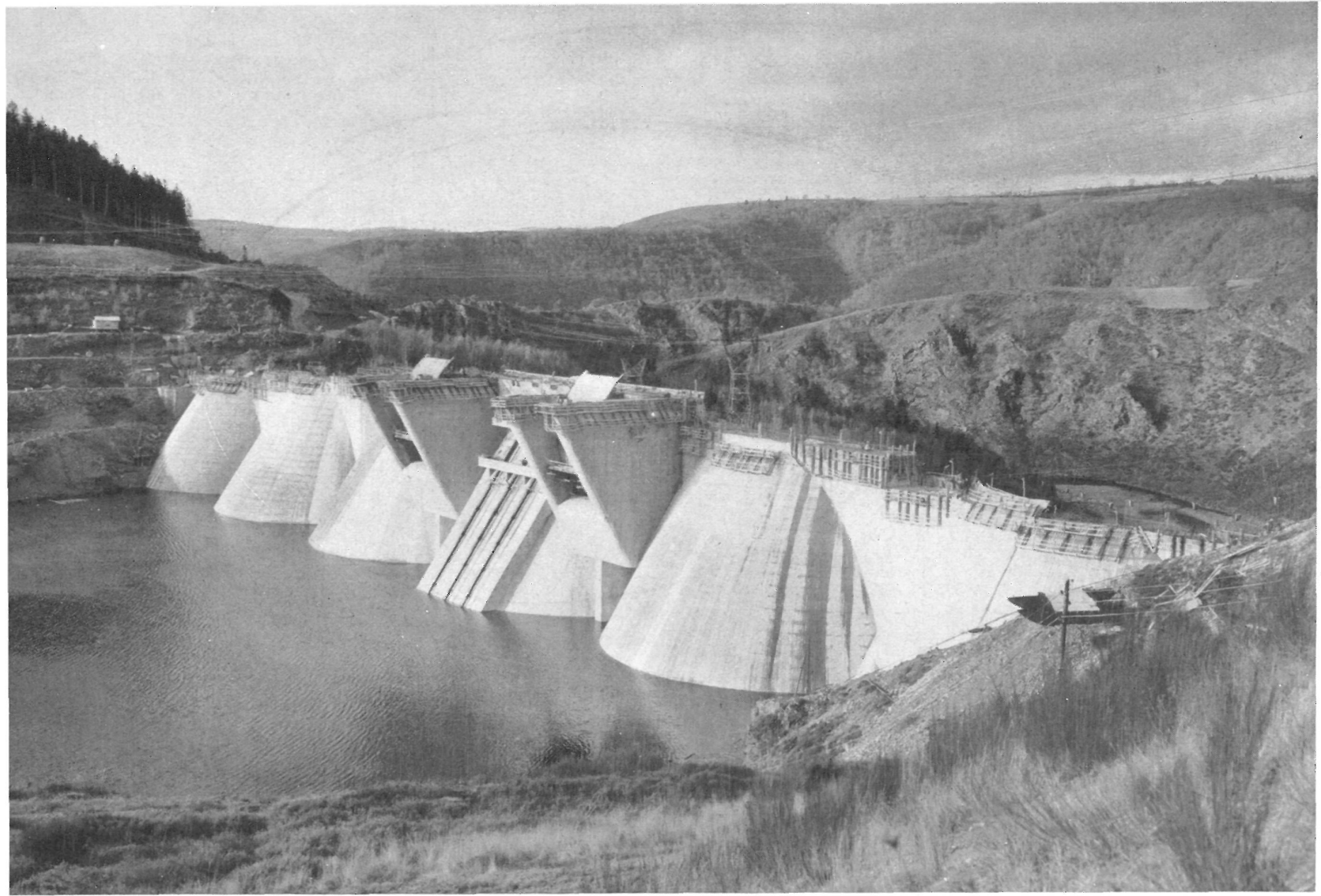

Fotos: H. BARANGER, PARIS

Además de los cálculos estáticos, para el dimensionado de contrafuertes, se ha estudiado el comportamiento de este nuevo tipo de presa utilizando modelos reducidos construidos con yeso.

La central y aliviaderos.-La central, de planta circular, se ha colocado entre los dos contrafuertes de mayor altura. Esta central dispone de dos grupos generadores de $34.000 \mathrm{~kW}$ cada uno, equipados con turbinas Francis, que utilizan un caudal de $53 \mathrm{~m} / \mathrm{s}$ y un salto medio útil de 58,50 metros.

La parte superior de los dos contrafuertes, entre los que se ha levantado la central, se ha aprovechado para formar en ella un aliviadero en forma de salto de esquí, en cuya parte superior se ha instalado una compuerta de sector de $13 \times 10 \mathrm{~m}$ para regulación del aliviadero (uno sobre cada contrafuerte).

La capacidad total de estos aliviaderos es de $1.900 \mathrm{~m}^{3}$, caudal que se ha estimado corresponde a las máximas avenidas posibles.

Ejecución de trabajos.-Las obras con carácter definitivo se empezaron en 1956. Anteriormente a esta fecha se abrió la galería provisional de derivación en la margen derecha. Esta galería tiene $400 \mathrm{~m}$ de longitud y $33 \mathrm{~m}{ }^{2}$ de sección, y ha sido calculada para un caudal de hasta $300 \mathrm{~m}^{3} / \mathrm{s}$. También fueron objeto de construcción anterior las ataguías que servirían para permitir cimentar la presa en seco.

La colocación de los $160.000 \mathrm{~m}^{3}$ de hormigón se terminó durante los primeros meses de 1960. Los áridos se han extraído de depósitos de aluviones del lecho del río Truyère. El hormigón se ha preparado en una central capaz de una producción de $80 \mathrm{~m}^{3} / \mathrm{h}$, y se ha colocado en obra utilizando tres blondines de $3,6 \mathrm{t}$ de capacidad y $634 \mathrm{~m}$ de vano. Con objeto de facilitar el hormigonado, a los cazos utilizados para el transporte del hormigón se les dio una forma especial, teniendo en cuenta el pequeño espesor relativo y la inclinación de los encofrados, así como la presencia de armaduras cuya densidad es de bastante consideración en la parte superior de la presa.

Cierre de embalse.-A partir del otoño de 1959, año en que se cerró la presa, inicia el almacenamiento de aguas, aunque los trabajos de hormigonado del resto de la obra no habían terminado en aquella fecha.

En marzo de 1960 se realizaron una serie de ensayos para comprobar el funcionamiento del desaguie de fondo y de los aliviaderos de superficie, lo que dio lugar a un lavado casi completo de los aluviones depositados en el lecho del río y en la parte de aguas abajo de la presa, desnudando la roca y excavando tres fosas de poca importancia, relativamente.

Aunque parece, según los ensayos, no existir peligro alguno para las obras en caso de erosión regresiva, se ha decidido adoptar, como precauciones suplementarias, la ejecución de una losa de hormigón protector, contra esta posible erosión, en la parte de aguas abajo, lo que evitará, a su vez, una ulterior degradación del lecho del río actualmente descarnado.

Los trabajos de la presa se dieron por terminados durante el verano de 1960, fecha en la cual también se terminó una carretera de $5,5 \mathrm{~m}$ de calzada que se apoya sobre la referida presa. La losa de hormigón que constituye la calzada de esta carretera vuela sobre la parte lateral de la presa en la zona de aguas arriba. Los elementos resistentes de apoyo de la calzada son de hormigón armado y del tipo prefabricado. 


\section{Le burngage de Gromdival}

C. Laroche et H. Legros, ingénieurs.

Cet important ouvrage est situé sur le tronçon supérieur de la Truyère, dans la région du Massif Central français.

La construction des barrages a été réalisée pour le compte de l'Electriticité de France. L'ensemble de l'aménagement hydroélectrique se compose de six barrages et d'autant de centrales qui utilisent une chute totale de $520 \mathrm{~m}$ de hauteur et les eaux contenues dans une retenue dont la capacité totale s'élève à $540 \times 10^{6} \mathrm{~m}^{3}$ qui assureront une production annuelle de $1.450 \times 10^{6} \mathrm{kWh}$.

Le barrage s'appuie sur un banc microschisteux qui, en plusieurs endroits, présente de vastes plis de grande puissance.

La digue de fermeture a une longueur totale de $330 \mathrm{~m}$ et $88 \mathrm{~m}$ de hauteur maximum, à partir de la base de fondations. L'épaisseur moyenne des voûtes est de $4,90 \mathrm{~m}$ à la base et de $1,50 \mathrm{~m}$ au couronnement.

La pente amont est de $0,70 \times 1$, avec la particularité que sa costruction n'a pas la forme cylindrique allongée jusqu'au couronnement, comme il est habituel dans ces cas, la partie supérieure de ces voûtes ayant été continuée en élévation, pour former une petite digue de gravité.

Le couronnement du barrage est rectiligne et supporte, en partie, la route en encorbellement qui le traverse.

Le barrage dispose d'un déversoir libre avec une vanne secteur et un déversoir en saut de ski sur chacun des deux contreforts entre lesquels la centrale a été construite.

\section{The Ereandival Dam}

C. Laroche \& H. Legros, engineers.

This important project is situated on the top section of the river Truyere, within the region of the French Central Massif. The dam has been constructed by the Electricite de France. The total hydroelectric development project in this zone involves six dams and six power stations, to exploit an overall hydrostatic head of $520 \mathrm{~ms}$. The water accumulated by these dams has a maximum volume of $540.10^{6}$ cubic metres, and will produce a yearly output of $1450.10^{6} \mathrm{kWh}$.

The Grandval dam rests on strong microschistous rock folds. Its length is $330 \mathrm{~ms}$, and its maximum height $88 \mathrm{~ms}$, measured from the foundation base. Structurally it is formed by a series of vaults supported by abutments, which are spaced at $50 \mathrm{~ms}$ intervals. The mean thickness of the vaults at the base is $4.90 \mathrm{~ms}$, and 1.50 at the crown. The vertical slope on the upstream side is 0.70 .

A feature of this design is that the vaults are not uniformly cylindrical up to the top, as is customary. The top part of the dam consists, in fact, of a small gravity dam, resting on the underlying vaulted structure.

The dam is straight at the top, and a road runs along it, on an overhanging structure.

The dam has a surface spillway, which is ski jump shaped, and runs over the two abutments on either side of the power station.

\section{Das Staumelhringm Grandral}

C. Laroche und H. Legros, Ingenieure.

Dieses bedeutende Werk liegt am Oberlauf des Truyère im Gebiete des französischen Zentralplateaus.

Die Stauwehre wurden auf Kosten der Electricité de France ausgeführt. Die Gesamtheit der hydroelektrischen Ausnutzung weist 6 Stauwehre und ebensoviele Werke auf, welche einen Wasserfall von $520 \mathrm{~m}$ Gesamthöhe und das in einem See gestaute Wasser ausnutzen, dessen Gesamtfassungvermögen auf $540 \times 10^{6} \mathrm{~m}^{3}$ ansteigt. Dies wird eine Jahreserzeugung von

Das Stauwehr stützt sich auf eine Schieferbank, die an einigen Stellen grosse Falten von ziemlicher Mächtigkeit aufweist. Der Abschlussdamm hat eine Gesamtlänge von $330 \mathrm{~m}$ und $88 \mathrm{~m}$ maximaler Höhe von der Zementbasis an. Seine Struktur wird von einer Reihe von Gewölben gebildet, die sich auf Widerlager stützen. Die lichte Weite zwischen ihnen beträgt $50 \mathrm{~m}$. Die mittlere Dicke der Gewölbe beträgt $4,90 \mathrm{~m}$ an der Basis und $1,50 \mathrm{~m}$ an der Krönung.

Die bergseitige Böschung beträgt $0,70 \times 1$ und hat die Besonderheit, dass ihr Bau keine bis zur Krönung reichende zylindrische Form aufweist, wie man meistens gewöhnt ist. Am oberen Teile dieser Gewölbe fuhr man fort, indem man erhöht einen kleinen Schwergewichtsstaudamm bildete.

Die Krönung des Stauwehres ist geradlinig. Sich darauf stützend verläuft eine Strasse, welche es in Auskragung überspannt.

Das Stauwehr verfügt über einen oberflächlichen Ueberlauf mit Sektorschütze und Talsperre in Form von Sprungschanzen über jedem der beiden Widerlager, zwischen denen das Zentralwerk erbaut worden ist. 